\title{
CHARACTERIZING SOME COMPLETELY REGULAR SEMIGROUPS BY THEIR SUBSEMIGROUPS
}

\author{
MARIO PETRICH \\ (Received 29 June 2011; accepted 29 October 2012; first published online 7 June 2013) \\ Communicated by M. G. Jackson
}

\begin{abstract}
We consider several familiar varieties of completely regular semigroups such as groups and completely simple semigroups. For each of them, we characterize their members in terms of absence of certain kinds of subsemigroups, as well as absence of certain divisors, and in terms of a homomorphism of a concrete semigroup into the semigroup itself. For each of these varieties $\mathcal{V}$ we determine minimal non- $\mathcal{V}$ varieties, provide a basis for their identities, determine their join and give a basis for its identities. Most of this is complete; one of the items missing is a basis for identities for minimal nonlocal orthogroups. Three tables and a figure illustrate the results obtained.
\end{abstract}

2010 Mathematics subject classification: 20M10.

Keywords and phrases: completely regular semigroup, subsemigroup, divisor, homomorphism, variety, minimal non- $\mathcal{V}$ variety, join, orthogroup, cryptogroup.

\section{Introduction and summary}

The seminal example of characterizing modular (respectively, distributive) lattices by the absence of one (respectively, two) types of sublattice has been emulated in the study of other algebraic systems. For such a class $C$ which is closed under taking of subalgebras, one arrives at 'forbidden subalgebras', namely those algebras which cannot occur as subalgebras of algebras in $C$. If the class of such forbidden objects is large, or unmanageable, and the class $C$ is also closed under homomorphic images, we may consider 'forbidden divisors', that is, algebras that do not occur as homomorphic images of subalgebras of members of $C$. All this, of course, up to isomorphism. One then searches for the class of all forbidden subalgebras or divisors, which may characterize the class $C$. We apply these ideas to some of the following classes of semigroups.

A completely regular semigroup $S$ (union of its subgroups) is enriched with the unary operation of inversion, that is, for any $a \in S, a^{-1}$ is the inverse of $a$ in the

(c) 2013 Australian Mathematical Publishing Association Inc. 1446-7887/2013 \$16.00 
maximal subgroup of $S$ containing $a$. This makes it possible to consider the class $C R$ of all such algebras as a variety.

Among the familiar subvarieties of $C \mathcal{R}$ are groups, completely simple semigroups, semilattices, semilattices of groups, cryptogroups (bands of groups), normal cryptogroups, orthogroups, and local orthogroups. Information on these varieties can be found in the book [6].

Some of the results for these varieties in the context of the first paragraph above are known. We complete the picture, with the exception of one case, by providing, for each of the above varieties, the following characterizations:

(a) a list of forbidden completely regular subsemigroups;

(b) a list of forbidden completely regular divisors;

(c) a semigroup whose homomorphic images determine the classes under study.

For each of these varieties $\mathcal{V}$ we list the varieties of completely regular semigroups minimal relative to being non- $\mathcal{V}$ and find their join.

One of these results generalizes the main theorem in [3]. The main tool for treating cryptogroups is the construction of all completely regular monoids with two generators accomplished in [4].

Section 2 covers terminology and notation, and includes a complete list of varieties which occur in the paper with bases for their identities. The varieties of completely simple semigroups, Clifford semigroups, semilattices, and normal cryptogroups are treated in Section 3. The next three sections contain a treatment of orthogroups, local orthogroups, and cryptogroups, respectively. These results are applied to groups, rectangular groups, normal orthogroups, orthocryptogroups, and local orthocryptogroups in Section 7. Section 8 consists of a review of the results in the paper in the form of three tables and a figure.

\section{Notation and terminology}

For our notation and terminology we follow the book [6] with a few exceptions noted below. For the convenience of the reader or for emphasis, we list the most important ones. Throughout the paper, $S$ denotes an arbitrary completely regular semigroup unless stated otherwise.

We work within the class $C \mathcal{R}$ of completely regular semigroups $S$ considered as unary semigroups relative to the operation $a \mapsto a^{-1}$, where for $a \in S, a^{-1}$ is the inverse of $a$ in the maximal subgroup of $S$ containing $a$. It follows that $C \mathcal{R}$ forms a variety, and thus all concepts on varieties apply to it. We will refer to varieties of completely regular semigroups simply as varieties. The lattice they form is denoted by $\mathcal{L}(C \mathcal{R})$.

In particular, if $\varnothing \neq \mathcal{X} \subseteq C \mathcal{R}$, then $\langle\mathcal{X}\rangle$ denotes the variety generated by $\mathcal{X}$. If $\mathcal{V} \in \mathcal{L}(C \mathcal{R})$ and the set $\left\{u_{\alpha}=v_{\alpha} \mid \alpha \in A\right\}$ forms a basis for identities valid in $\mathcal{V}$, we write $\mathcal{V}=\left[u_{\alpha}=v_{\alpha}\right]_{\alpha \in A}$. If $\mathcal{U}, \mathcal{V} \in \mathcal{L}(C \mathcal{R})$ are such that $\mathcal{U} \nsubseteq \mathcal{V}$, we say that $\mathcal{U}$ is a non- $\mathcal{V}$ variety.

There are some special semigroups which occur often in this context:

$$
L_{2}=\left\{\ell_{1}, \ell_{2}\right\}, \quad R_{2}=\left\{r_{1}, r_{2}\right\}, \quad Y_{2}=\{0,1\}
$$


with left zero, right zero, and the usual multiplication, respectively. The semigroups $L G_{n}$ and $R G_{n}$ are constructed in [6, page 238]; we will provide an alternative construction of these semigroups in Lemma 6.1.

By $E(X)$ we denote the set of all idempotents of a subset $X$ of a semigroup. If $S$ is a semigroup with identity, we write $S=S^{1}$; otherwise $S^{1}$ is $S$ with an identity adjoined. If $T$ is a semigroup which is not isomorphic to a subsemigroup of $S$, we say that $T$ is a forbidden subsemigroup for $S$. A semigroup $T$ is a divisor of $S$ if $T$ is a homomorphic image of a subsemigroup of $S$. If $T$ is not isomorphic to a divisor of $S$, then $T$ is a forbidden divisor for $S$. Since we are dealing only with completely regular semigroups, any subsemigroup or divisor or semigroup generated by a set will be tacitly assumed to be completely regular, occasionally specified as such for emphasis.

For $a \leq b$ in a lattice $L,[a, b]$ denotes the interval with lower end $a$ and upper end $b$.

In the list below, we denote varieties by their standard acronyms, give bases for their identities, and in a few cases, their generators. These varieties occur frequently in the text, sometimes as parts of intersections. Hence the bases listed provide bases for other varieties. Most of these bases can be found in [6] and we list their exact references whenever possible. This makes it feasible to state only the acronyms; their bases can be readily read off from this list. Recall that $L$ stands for the local operator.

Unlike in [6], in composite notation we write $\mathcal{A}$ instead of $H \mathcal{A}$; also we write $\operatorname{Rec} \mathcal{A}$ for rectangular abelian groups while in [6] they are denoted by $\operatorname{Re} \mathcal{A}$. The letter $p$ always stands for a prime; let $\mathbb{P}$ denote the set of all positive prime integers. A rectangular band isomorphic to $L \times R$ where $L \in \mathcal{L} \mathcal{Z}$ and $R \in \mathcal{R Z}$ with $|L|,|R| \leq 2$ we call small.

Glossary We do not list duals. The references generally contain more information about these varieties.

$$
\begin{aligned}
& \mathcal{L} \mathcal{Z}=[a=a x]=\left\langle L_{2}\right\rangle, \\
& \mathcal{R B}=[a=a x a]=\left\langle L_{2} \times R_{2}\right\rangle, \\
& \mathcal{S}=\left[a x=x^{0} a\right]=\left\langle Y_{2}\right\rangle, \\
& \mathcal{N B}=\left[\text { axya }=a^{0} y x a\right]=\left\langle L_{2} \times Y_{2} \times R_{2}\right\rangle, \\
& \mathcal{L} \mathcal{R B}=[\text { ax }=\text { axa }]=\left\langle L_{2}^{1}\right\rangle, \\
& \mathcal{R e} \mathcal{B}=[\text { axya }=\text { axaya }]=\left\langle\left(L_{2} \times R_{2}\right)^{1}\right\rangle, \quad \text { see [6, Theorem V.1.9], } \\
& \mathcal{B}=\left[a=a^{0}\right] . \\
& \mathcal{G}=\left[a^{0}=b^{0}\right], \\
& \operatorname{ReG}=\left[a^{0}=a^{0} x^{0} a^{0}\right], \quad \text { see [6, Corollary III.5.3], } \\
& C \mathcal{S}=\left[a^{0}=(a x a)^{0}\right], \quad \text { see [6, Proposition III.1.1], } \\
& \mathcal{S G}=\left[a x^{0}=x^{0} a\right], \quad \text { see [6, Theorem IV.2.4], }
\end{aligned}
$$




$$
\begin{aligned}
& \mathcal{N B G}=\left[(\text { axya })^{0}=(\text { ayxa })^{0}\right], \\
& \text { see [6, Theorem IV.1.6], } \\
& \mathcal{R B G}=\left[(\text { axya })^{0}=(\text { axaya })^{0}\right], \\
& \mathcal{B G}=\left[(a b)^{0}=\left(a^{0} b^{0}\right)^{0}\right], \\
& \mathcal{N O}=\left[a x y^{0} a=a y^{0} x a\right], \\
& \mathcal{R O}=\left[a x y a=a x a^{0} y a\right], \\
& \mathcal{O}=\left[a^{0} b^{0}=\left(a^{0} b^{0}\right)^{0}\right], \\
& L O=\left[(a x)^{0}(b x)^{0}=\left((a x)^{0}(a y)^{0}\right)^{0}\right], \\
& O \mathcal{B G}=\left[a^{0} b^{0}=(a b)^{0}\right], \\
& L O \mathcal{B G}=\left[(a x)^{0}(a y)^{0}=(a x a y)^{0}\right], \\
& \operatorname{Rec} \mathcal{A}=\left[a^{0}=\operatorname{axya}(\text { ayxa })^{-1}\right], \\
& C \mathcal{S} \mathcal{A}=\left[a^{0}=a^{0} x a\left(a x a^{0}\right)^{-1}\right]=\left\langle D_{\infty}\right\rangle, \\
& \mathcal{N B \mathcal { A }}=[\text { axaya }=\text { ayaxa }], \\
& \mathcal{L} \mathcal{R O} \mathcal{A}=\left[(a x a)^{2} x a y=a x(a x a)^{2} y a^{0}\right], \\
& \mathcal{R O A}=\left[b(a x a)^{0} x a y b=\operatorname{bax}(a x a)^{0} b^{0} y b\right], \\
& H \mathcal{A}=\left[\operatorname{ax}(a x a)^{0}=(a x a)^{0} x a\right], \\
& \text { see [6, Proposition V.4.4], } \\
& \text { see [6, Theorem II.8.1]. } \\
& \text { see [6, Theorem IV.2.7], } \\
& \text { see [6, Theorem V.3.3], } \\
& \text { see [6, Theorem II.5.3], } \\
& \text { see [6, Corollary II.7.5], } \\
& \text { see [6, Theorem II.8.5], } \\
& \text { see [6, Corollary II.8.6]. }
\end{aligned}
$$

For any prime $p$ :

$$
\begin{aligned}
\mathcal{A}_{p} & =\left[a^{p}=x^{0} a^{-1} x^{-1} a x\right]=\left\langle\mathbb{Z}_{p}\right\rangle, & & \text { see [2, Theorem 6.1(i) }], \\
\mathcal{C S} \mathcal{A}_{p} & =\left[a^{p}=a x\left(a^{0} x a\right)^{-1}\right]=\left\langle D_{p}\right\rangle=\mathcal{D}_{p}, & & \text { see [2, Theorem 6.4(i) }], \\
\mathcal{L} \mathcal{R O A} \mathcal{A}_{p} & =\left[(a x a)^{2} x a y=a x(a x a)^{2} y a^{p}\right]=\left\langle L G_{p}\right\rangle, & & \text { see [2, Theorem 6.4(v)]. }
\end{aligned}
$$

This glossary will be used consistently. Combining these identities will yield bases for identities for most varieties appearing in the paper.

\section{Varieties $C \mathcal{S}, \mathcal{S G}, \mathcal{S}$, and $\mathcal{N B G}$}

We first provide a multiple characterization of members of each of these varieties. This is followed by the determination of minimal non- $\mathcal{V}$ varieties and of their join. These are simple statements which are either well known or easy to prove, so we move quickly.

It is useful to keep in mind the following lemma.

Lemma 3.1. Let $\mathcal{V} \in \mathcal{L}(C \mathcal{R})$ and $\mathbf{U} \subseteq \mathcal{L}(C \mathcal{R})$ be such that each member of $\mathbf{U}$ is a minimal non- $\mathcal{V}$ variety and every non- $\mathcal{V}$ variety contains a member of $\mathbf{U}$. Then $\mathbf{U}$ is the set of all minimal non- $\mathcal{V}$ varieties.

Proof. Let $\mathcal{W}$ be a minimal non- $\mathcal{V}$ variety. Then there exists $\mathcal{U} \in \mathbf{U}$ such that $\mathcal{U} \subseteq \mathcal{W}$, which by minimality of $\mathcal{W}$ implies that $\mathcal{W}=\mathcal{U} \in \mathbf{U}$. 
Proposition 3.2. The following conditions on $S$ are equivalent.

(i) $S \in C \mathcal{S}$.

(ii) $S$ has no subsemigroups isomorphic to $Y_{2}$.

(iii) $S$ has no divisors isomorphic to $Y_{2}$.

(iv) If $\chi: Y_{2} \rightarrow S$ is a homomorphism, then the image of $\chi$ is trivial.

PRoof. Part of this is well known and the rest requires a straightforward argument.

Proposition 3.3. The following conditions on $S$ are equivalent.

(i) $S \in \mathcal{S}$.

(ii) $S$ has no subsemigroups isomorphic to $L_{2}, R_{2}, \mathbb{Z}$, or $\mathbb{Z}_{n}$ for any $n>1$.

(iii) $S$ has no divisors isomorphic to $L_{2}, R_{2}$, or $\mathbb{Z}_{p}$ for a prime $p$.

(iv) If $\chi: L_{2} \times \mathbb{Z} \times R_{2} \rightarrow S$ is a homomorphism, then the image of $\chi$ is trivial.

Proof. Straightforward.

Proposition 3.4. The following conditions on $S$ are equivalent.

(i) $S \in \mathcal{S G}$.

(ii) $S$ has no subsemigroups isomorphic to $L_{2}$ or $R_{2}$.

(iii) $S$ has no divisors isomorphic to $L_{2}$ or $R_{2}$.

(iv) If $\chi: L_{2} \times R_{2} \rightarrow S$ is a homomorphism, then the image of $\chi$ is trivial.

Proof. Straightforward.

Proposition 3.5. The following conditions on $S$ are equivalent.

(i) $S \in \mathcal{N} \mathcal{B G}$.

(ii) $S$ has no subsemigroups isomorphic to $L_{2}^{1}$ or $R_{2}^{1}$.

(iii) $S$ has no divisors isomorphic to $L_{2}^{1}$ or $R_{2}^{1}$.

(iv) If $\chi:\left(L_{2} \times R_{2}\right)^{1} \rightarrow S$ is a homomorphism, then the image of $\chi$ is a one-or twoelement semilattice.

Proof. The equivalence of (i), (ii) and (iii) and the forward implication from these to (iv) is a direct consequence of [6, Theorem IV.1.6].

Now we prove that (iv) implies (ii). If $S$ had a subsemigroup $T$ isomorphic to $L_{2}^{1}$, then $T$ would be a homomorphic image of $\left(L_{2} \times R_{2}\right)^{1}$, contrary to the hypothesis. The same goes for $R_{2}^{1}$.

For the four varieties $\mathcal{V}$ studied in this section, we now provide information regarding minimal non- $\mathcal{V}$ varieties.

THeorem 3.6. (i) $\mathcal{S}$ is a minimal non-CS variety. Every non-CS variety contains $\mathcal{S}$.

(ii) $\mathcal{L} Z$ and $\mathcal{R Z}$ are minimal non-SG varieties. Every non-SG variety contains either $\mathcal{L} \mathcal{Z}$ or $\mathcal{R Z}$. Moreover, $\mathcal{R B}$ is the join of all minimal non-SG varieties.

(iii) $\mathcal{L} \mathcal{Z}, \mathcal{R Z}$, and $\mathcal{A}_{p}$ for p prime are minimal non-S varieties. Every non-S variety contains at least one of $\mathcal{L} \mathcal{Z}, \mathcal{R Z}$, or $\mathcal{A}_{p}$ for p prime. Moreover, $\operatorname{Rec} \mathcal{A}$ is the join of all minimal non-S varieties. 
(iv) $\mathcal{L} \mathcal{R}$ and $\mathcal{R R B}$ are minimal non- $\mathcal{N B G}$ varieties. Every non- $\mathcal{N} \mathcal{B G}$ variety contains either $\mathcal{L} \mathcal{R B}$ or $\mathcal{R} \mathcal{R}$. Moreover, $\mathcal{R} \mathcal{B}$ is the join of all minimal non$\mathcal{N B G}$ varieties.

Proof. Parts (i) and (ii) require a simple argument. In part (iii), it should be observed that $\mathcal{L} \mathcal{Z}, \mathcal{S}, \mathcal{R Z}$, and $\mathcal{A}_{p}$ are the atoms of $\mathcal{L}(C \mathcal{R})$ and that $\bigvee_{p} \mathcal{A}_{p}=\mathcal{A}$, the variety of abelian groups.

(iv) It is well known that $\mathcal{L R B}, \mathcal{R} \mathcal{R} \nsubseteq \mathcal{N} \mathcal{B G}$ and that $\mathcal{L} \mathcal{R} \vee \mathcal{R} \mathcal{B}=\mathcal{R} e \mathcal{B}$. That all proper subvarieties of $\mathcal{L} \mathcal{R B}$ and $\mathcal{R} \mathcal{R} B$ are minimal follows from [6, Theorem V.1.7]. Hence $\mathcal{L} \mathcal{R} B$ and $\mathcal{R} \mathcal{B}$ are minimal non- $\mathcal{N B G}$ varieties. Let $\mathcal{V}$ be a non- $\mathcal{N} \mathcal{B} G$ variety and $S \notin \mathcal{N B G}$. In view of Proposition 3.5, $S$ must contain an isomorphic copy of either $L_{2}^{1}$ or $R_{2}^{1}$. By [6, Lemma V.1.4] and its dual, either $\mathcal{L} \mathcal{R} \subseteq \mathcal{V}$ or $\mathcal{R} \mathcal{R} \subseteq \mathcal{V}$.

\section{Variety $O$}

Here we follow the pattern of the preceding section for the variety $O$ of orthogroups. The reference [2, Section 3] contains further information for this case; we adopt its notation as follows.

For $n>1$, let

$$
\begin{aligned}
& D_{n}=\mathcal{M}\left(\{0,1\}, \mathbb{Z}_{n},\{0,1\} ;\left[\begin{array}{ll}
\overline{0} & \overline{0} \\
\overline{0} & \overline{1}
\end{array}\right]\right), \\
& D_{\infty}=\mathcal{M}\left(\{0,1\}, \mathbb{Z},\{0,1\} ;\left[\begin{array}{ll}
0 & 0 \\
0 & 1
\end{array}\right]\right) .
\end{aligned}
$$

These semigroups play a central role in [2] for the characterization of minimal non- $O$ varieties. We start with homomorphic images of $D_{\infty}$.

Lemma 4.1. A semigroup $S$ is a homomorphic image of $D_{\infty}$ if and only if $S$ is isomorphic to one of the following semigroups: $D_{\infty}, D_{n}$ for $n>1$, small rectangular bands.

Proof. Necessity. Let $\rho$ be a congruence on $D_{\infty}$. By [6, Theorem III.4.6], we have $\rho=\rho_{(r, N, \pi)}$ for some admissible triple $(r, N, \pi)$; see also [6, Lemma III.4.4]. Here $r$ and $\pi$ are equivalence relations on the set $\{0,1\}$ and $N$ is a subgroup of $\mathbb{Z}$ satisfying the conditions

$$
\begin{aligned}
& i r j \Longrightarrow p_{k i}-p_{k j} \in N \text { for all } k \in\{0,1\} \\
& i \pi j \Longrightarrow p_{i k}-p_{j k} \in N \text { for all } k \in\{0,1\}
\end{aligned}
$$

We distinguish the following cases.

Case 1. $r=\pi=\varepsilon$. Now conditions (4.1) and (4.2) are vacuous and $S / \rho$ is isomorphic either to $D_{\infty}$, or to $D_{n}$ for some $n>1$, or to $L_{2} \times R_{2}$.

Case 2. $r=\omega, \pi=\varepsilon$. Condition (4.2) yields $p_{12}-p_{22} \in N$, so that $-1 \in N$ which implies that $N=\mathbb{Z}$. We thus arrive at $S / \rho \cong R_{2}$. 
Case 3. $r=\varepsilon, \pi=\omega$. This is dual to Case 2. Hence $S / \rho \cong L_{2}$.

Case 4. $r=\pi=\omega$. The same type of argument yields $\rho=\omega$ and thus $S / \rho$ is a trivial semigroup.

Sufficiency. A straightforward argument shows that each of the semigroups listed is a homomorphic image of $D_{\infty}$.

THEOREM 4.2. The following conditions on $S$ are equivalent.

(i) $S \in O$.

(ii) $S$ has no subsemigroups isomorphic to $D_{\infty}$ or to $D_{n}$ for any $n>1$.

(iii) $S$ has no divisors isomorphic to $D_{p}$ for any prime $p$.

(iv) If $\chi: D_{\infty} \rightarrow S$ is a homomorphism, then the image of $\chi$ is a small rectangular band.

Proof. (i) implies (ii). In $D_{\infty}, e=(0,0,1)$ and $f=(1,0,0)$ are idempotents but $e f$ is not. Similarly for $D_{n}$.

(ii) implies (iii). By [6, Corollary III.5.5], $S$ must be orthodox. Hence all its divisors are orthodox as well. Since none of $D_{n}$ and $D_{\infty}$ is orthodox, they cannot be divisors of $S$.

(iii) implies (iv). This follows directly from Lemma 4.1 and [2, Lemma 3.2].

(iv) implies (i). By contrapositive, assume that $S$ is not orthodox. By [6, Corollary III.5.5], $S$ has a subsemigroup isomorphic to $D_{n}$ for some $n>1$ or to $D_{\infty}$. By Lemma 4.1, $D_{n}$ is a homomorphic image of $D_{\infty}$ and is not a rectangular band.

In order to handle minimal non- $O$ varieties, we need the following lemma.

LEMMA 4.3. For every $n \in \mathbb{Z}$ and every prime $p$, define $n_{p}$ by $n=p q+n_{p}$ for some $q \in \mathbb{Z}$ with $0 \leq n_{p}<p$, and let $\left[n_{p}\right]_{p}$ be the corresponding element of $\mathbb{Z}_{p}$. The mapping

$$
\chi: n \longrightarrow\left(\left[n_{p}\right]_{p}\right)_{p \in \mathbb{P}} \quad(n \in \mathbb{Z})
$$

is an embedding of $\mathbb{Z}$ into $\prod_{p} \mathbb{Z}_{p}$.

Proof. Straightforward.

THeOREM 4.4. For every prime $p$, let $\mathcal{D}_{p}=\left\langle D_{p}\right\rangle$ and $\mathcal{D}_{\infty}=\left\langle D_{\infty}\right\rangle$.

(i) $\mathcal{D}_{p}=C S \mathcal{A}_{p}$.

(ii) $\mathcal{D}_{p}$ is a minimal non- $O$ variety.

(iii) Every non-O variety contains $\mathcal{D}_{p}$ for some prime $p$.

(iv) If $\mathcal{D}_{p}=\mathcal{D}_{q}$ for some primes $p$ and $q$, then $p=q$.

(v) $\mathcal{D}_{\infty}=\bigvee_{p} \mathcal{D}_{p}=C \mathcal{S A}$.

Proof. Part (i) was proved in [2, Lemma 4.7], while parts (ii) and (iii) follow from [6, Corollary III.5.5].

(iv) Groups in $\mathcal{D}_{p}$ are abelian with exponents dividing $p$. Hence if $\mathcal{D}_{p}=\mathcal{D}_{q}$, then $p$ divides $q$ and $q$ divides $p$ so that $p=q$. 
(v) Define a mapping by

$$
\varphi:(i, n, j) \longrightarrow(i, n \chi, j) \quad\left((i, n, j) \in D_{\infty}\right)
$$

where $\chi$ was defined in Lemma 4.3. By this lemma and in view of the form of the sandwich matrices of $D_{\infty}$ and $D_{p}$, we deduce that $\varphi$ embeds $D_{\infty}$ into $\prod_{p} D_{p}$. It follows that $\mathcal{D}_{\infty} \subseteq \bigvee_{p} \mathcal{D}_{p}$. The opposite inclusion follows from Lemma 4.3.

Since $D_{\infty} \in C \mathcal{S A}$, we deduce that $\mathcal{D}_{\infty} \subseteq C \mathcal{S A}$. From the list of overabelian completely simple semigroups in [6, Theorem VIII.9.3], we see that $D_{\infty}$ is contained only in $C \mathcal{S A}$, which shows that $D_{\infty}$ is not contained in any proper subvariety of $C \mathcal{S A}$ and thus $\mathcal{D}_{\infty}=C \mathcal{S A}$.

If $S \in C \mathcal{S A}$, then by [6, Proposition III.6.3], $S$ satisfies the identity $a^{0} x a=a x a^{0}$, whence

$$
a x\left(a^{0} x a\right)^{-1}=\left(a x a^{0}\right)\left(a^{0} x a\right)^{-1}=\left(a^{0} x a\right)^{0}=a^{0} .
$$

Conversely, if $S$ satisfies the identity $a^{0}=a x\left(a^{0} x a\right)^{-1}$, then $S$ is completely simple and its subgroups are abelian.

\section{Variety $L O$}

We continue with the same pattern. Locally orthogroup varieties were studied in [2, Section 4]. In particular, [2, Corollary 4.6], where unfortunately the word 'non' is missing, provides the complete set of minimal non- $L O$ varieties. Also the variety at the very top of [2, Diagram 2] should be $\mathcal{D}_{p}^{1}$ and not $\mathcal{D}_{p}$.

Lemma 5.1. A semigroup $S$ is a homomorphic image of $D_{\infty}^{1}$ if and only if $S$ is isomorphic to one of the semigroups in Lemma 4.1 with an identity adjoined or is trivial.

Proof. Necessity. Let $\rho$ be a congruence on $D_{\infty}^{1}$. We consider two cases.

Case 1. $1 \rho(i, n, j)$ for some $(i, n, j) \in D_{\infty}$. By [6, Theorem III.4.6], we have $\left.\rho\right|_{D_{\infty}}=\rho_{(r, N, \pi)}$ for an admissible triple $(r, N, \pi)$. Since $S / \rho$ is a monoid, it follows that $r=\pi=\omega$, which as in the proof of Lemma 4.1 implies that $N=\mathbb{Z}$. It follows that $\rho=\omega$ and $S / \rho$ is a trivial semigroup.

Case 2. $\{1\}$ is a $\rho$-class. In view of Lemma 4.1 , we conclude that $D_{\infty} /\left(\left.\rho\right|_{D_{\infty}}\right)$ is isomorphic to one of the semigroups in that lemma. But then $D_{\infty} / \rho$ is isomorphic to one of the semigroups in that lemma with an identity adjoined.

Sufficiency. This requires a straightforward argument.

THEOREM 5.2. The following conditions on $S$ are equivalent.

(i) $S \in L O$.

(ii) $S$ has no subsemigroups isomorphic to $D_{\infty}^{1}$ or to $D_{n}^{1}$ for any $n>1$.

(iii) $S$ has no divisors isomorphic to $D_{p}^{1}$ for any prime $p$.

(iv) If $\chi: D_{\infty}^{1} \rightarrow S$ is a nontrivial homomorphism, then the image of $\chi$ is a small rectangular band with an identity adjoined. 
Proof. (i) implies (ii). See the proof of Theorem 4.2.

(ii) implies (i). By contrapositive, assume that $S$ is not locally orthodox. Then there exists $e \in E(S)$ such that $e S e$ is not orthodox. By Theorem 4.2, eSe contains a subsemigroup $T$ isomorphic either to $D_{\infty}$ or to $D_{n}$ for some $n>1$. Clearly $e \notin T$, so that $T \cup\{e\}$ must be isomorphic either to $D_{\infty}^{1}$ or to $D_{n}^{1}$.

(i) implies (iii). Since the class of all locally orthogroups is a variety and neither $D_{\infty}^{1}$ nor $D_{n}^{1}$ is locally orthodox, $S$ cannot have divisors isomorphic to $D_{\infty}^{1}$ or to $D_{n}^{1}$ for any $n>1$.

(iii) implies (iv). This follows from Lemma 5.1 and [2, Lemma 4.2].

(iv) implies (i). The argument here is similar to that in '(ii) implies (i)' above.

THEOREM 5.3. For every prime $p$, let $\mathcal{D}_{p}^{1}=\left\langle D_{p}^{1}\right\rangle$ and $\mathcal{D}_{\infty}^{1}=\left\langle D_{\infty}^{1}\right\rangle$.

(i) $\mathcal{D}_{p}^{1}$ is a minimal non- $L O$ variety.

(ii) Every non- $L O$ variety contains $\mathcal{D}_{p}^{1}$ for some prime $p$.

(iii) If $\mathcal{D}_{p}^{1}=\mathcal{D}_{q}^{1}$ for some primes $p$ and $q$, then $p=q$.

(iv) $\mathcal{D}_{\infty}^{1}=\bigvee_{p} \mathcal{D}_{p}^{1}$.

Proof. Parts (i) and (ii) were proved in [2, Theorem 4.5], while the argument for part (iii) is the same as in Theorem 4.4(iv).

(iv) We have seen in the proof of Theorem 4.4 that $D_{\infty}$ can be embedded into $\prod_{p} D_{p}$. In $\prod_{p} D_{p}^{1}$, the identity element has all coordinates equal to 1 , so we may embed $D_{\infty}^{1}$ into $\prod_{p} D_{p}^{1}$ by mapping 1 to $(1)_{p}$, and the rest of the elements of $D_{\infty}^{1}$ as for $D_{\infty}$. This gives $\mathcal{D}_{\infty}^{1} \subseteq \bigvee_{p} \mathcal{D}_{p}^{1}$.

Since $D_{p}$ is a homomorphic image of $D_{\infty}$, it follows easily that $D_{p}^{1}$ is a homomorphic image of $D_{\infty}^{1}$. But then $\mathcal{D}_{p}^{1} \subseteq \mathcal{D}_{\infty}^{1}$ for all primes $p$, and thus $\bigvee_{p} \mathcal{D}_{p}^{1} \subseteq \mathcal{D}_{\infty}^{1}$.

In [2] we did not have a basis for $\mathcal{D}_{p}^{1}$. In this paper also a basis for $\mathcal{D}_{\infty}^{1}$ is missing.

\section{Variety $\mathcal{B} G$}

We continue here with the pattern of the preceding three sections, now for cryptogroups (bands of groups). Our statements and notation rely heavily on the paper [4] where arbitrary completely regular monoids with two generators were constructed. We start with the devices needed from that paper.

From [4, Construction 3.1] we borrow the following. Let $G$ be a group generated by the set $\left\{g_{n} \mid n \in \mathbb{Z}\right\}$, where we allow that $g_{m}=g_{n}$ if $m \neq n$. Let $P=\left(p_{n m}\right)$ be a $\mathbb{Z} \times \mathbb{Z}$ matrix with

$$
p_{n m}= \begin{cases}g_{0}^{-1} \cdots g_{n-1}^{-1} g_{m+n-1} \cdots g_{m} & \text { if } n>0 \\ 1 & \text { if } n=0 \\ g_{-1} \cdots g_{n} g_{m+n}^{-1} \cdots g_{m-1}^{-1} & \text { if } n<0\end{cases}
$$

and let

$$
\Gamma^{*}=\mathcal{M}(\mathbb{Z}, G, \mathbb{Z} ; P)
$$


Note that $P$ is normalized at 0 . Let $\theta=(\alpha, \varphi ; \psi, \beta)$ where

$$
\alpha n=n+1, \quad \varphi n=g_{n}, \quad n \psi=p_{n 1} g_{0}, \quad n \beta=n+1 \quad(n \in \mathbb{Z}) .
$$

We will now specialize the construction in [4] to the present situation. For reasons of economy, we will use the material in that paper without repetition except for the following. Let $\bar{\Omega}\left(\Gamma^{*}\right)$ be the translational hull of $\Gamma^{*}$ and

$\Omega\left(\Gamma^{*}\right)=\left\{(\alpha, \varphi ; \psi, \beta) \in(\mathcal{T}(\mathbb{Z}) \mathrm{wl} G) \times\left(G \mathrm{wr} \mathcal{T}^{\prime}(\mathbb{Z})\right) \mid(n \psi) p_{n \beta, m}=p_{n, \alpha m}(\varphi m), m, n \in \mathbb{Z}\right\}$

and note that $\bar{\Omega}\left(\Gamma^{*}\right) \cong \Omega\left(\Gamma^{*}\right)$.

By [4, Lemma 3.2], $\theta$ is in the group of units of $\Omega\left(\Gamma^{*}\right)$. Next we follow the notation introduced before Lemma 3.4 of [4]. Let $B=\langle\theta\rangle$, the (cyclic) group generated by $\theta$, and $B^{0}$ be $B$ with a zero adjoined. Further, let $\Gamma$ be the ideal extension of $\Gamma^{*}$ by $B^{0}$ determined by the action of $\theta$, that is, by $\theta^{k}$ for $k \in \mathbb{Z}$.

We now modify the above construction; see [4, Section 4]. Let $\ell>0$ be an integer and let

$$
I_{\ell}=\{0, \ldots, \ell-1\}, \quad G_{\ell}=\left\langle g_{0}, \ldots, g_{\ell-1}\right\rangle .
$$

Extend the sequence $g_{0}, \ldots, g_{\ell-1}$ to the sequence $\left\{g_{n} \mid n \in \mathbb{Z}\right\}$ with the proviso that

$$
g_{m}=g_{n} \Longleftrightarrow m \equiv n \quad(\bmod \ell) \text {. }
$$

Now let

$$
\begin{aligned}
\Gamma_{\ell}^{*} & =\mathcal{M}\left(I_{\ell}, G_{\ell}, \mathbb{Z} ;\left.P\right|_{\mathbb{Z} \times I_{\ell}}\right), \\
\alpha_{\ell} & =(0, \ldots, \ell-1) \text { be a cycle, } \\
\theta_{\ell} & =\left(\alpha_{\ell},\left.\varphi\right|_{I_{\ell}} ; \psi, \beta\right) .
\end{aligned}
$$

By [4, Lemma 4.1], $\theta_{\ell}$ is in the group of units of $\Omega\left(\Gamma_{\ell}^{*}\right)$. Denote by $\Gamma_{\ell}$ the ideal extension of $\Gamma_{\ell}^{*}$ by $\left\langle\theta_{\ell}\right\rangle^{0}$ determined by $\theta_{\ell}$, that is, by $\theta_{\ell}^{k}$ for $k \in \mathbb{Z}$.

We denote by $\Gamma_{\ell}^{\prime}$ the (left-right) dual of $\Gamma_{\ell}$.

Next we let $r>0$ be an integer, and

$$
\Lambda_{r}=\{0, \ldots, r-1\}, \quad \text { and } \quad \beta_{r}=(0, \ldots, r-1) \text { be a cycle. }
$$

We extend the sequence $g_{0}, \ldots, g_{r-1}$ to $\left\{g_{n} \mid n \in \mathbb{Z}\right\}$ as above. In addition, we impose the condition

(C) $g_{r-1} \cdots g_{0}=g_{m+r-1} \cdots g_{m} \quad(m=1, \ldots, \ell-1)$.

Let $\gamma$ be the congruence on $G_{\ell}$ induced by condition (C) and let $G_{\ell r}=G_{\ell} / \gamma$. Define

$$
\begin{aligned}
\Gamma_{\ell r}^{*} & =\mathcal{M}\left(I_{\ell}, G_{\ell r} ; \Lambda_{r} ;\left.P\right|_{\Lambda_{r} \times I_{\ell}}\right), \\
\theta_{\ell r} & =\left(\alpha_{\ell},\left.\varphi\right|_{I_{\ell}} ;\left.\psi\right|_{\Lambda_{r}}, \beta_{r}\right) .
\end{aligned}
$$

By [4, Lemma 5.1], $\theta_{\ell r}$ is in the group of units of $\Omega\left(\Gamma_{\ell r}^{*}\right)$. Let $\Gamma_{\ell r}$ be the ideal extension of $\Gamma_{\ell r}^{*}$ by $\left\langle\theta_{\ell r}\right\rangle^{0}$ determined by $\theta_{\ell r}$, that is, by $\theta_{\ell r}^{k}$ for $k \in \mathbb{Z}$. 
Now let $B$ be a cyclic group of order $s$ (finite or infinite) generated by an element $b$. If $s>0$, we assume that for some positive integers $p$ and $q$,

$$
s=p \ell=q r, \quad\left(g_{\ell-1} \cdots g_{0}\right)^{p}=\left(g_{r-1} \cdots g_{0}\right)^{q}=1 .
$$

Let $\tau: B \rightarrow\left\langle\theta_{\ell r}\right\rangle$ be the homomorphism induced by the mapping $b \mapsto \theta_{\ell r}$. Then $\tau$ produces an ideal extension $\Gamma_{\ell r s}$ of $\Gamma_{\ell r}^{*}$ by $B^{0}$.

We now construct a special case of $\Gamma$ as follows; see [4, Section 6]. Let $H=\left\{h_{n} \mid\right.$ $n \in \mathbb{Z}\}$ where $h_{m}=h_{n}$ only if $m=n$. Denote by $F H$ the free group on $H$. We specify the parameters and notation of $\Gamma$ as

$$
\begin{gathered}
G \longrightarrow F H, \quad \Gamma \longrightarrow \Gamma_{\infty}, \quad \Gamma^{*} \longrightarrow \Gamma_{\infty}^{*}, \\
\theta=(\alpha, \varphi ; \psi, \beta) \longrightarrow \kappa=(\xi, \sigma ; \tau, \eta), \\
g_{n} \longrightarrow h_{n}, \quad p_{n m} \longrightarrow q_{n m},
\end{gathered}
$$

with all the formulae for $\Gamma$ retaining their meaning in the new notation. For details and results concerning the above concepts and notation, consult [4].

Recall that the semigroups $L G_{n}$ and $R G_{n}$ were constructed in [6, page 238]. Next we give an explicit characterization of $L G_{n}$; the semigroup $R G_{n}$ can be constructed in a dual manner.

LeMma 6.1. Let $n>1$.

(i) Denote by $L_{n}$ the left zero semigroup on the set $\{0, \ldots, n-1\}$. On $L_{n} \cup \mathbb{Z}_{n}$ define a multiplication such that the operations in $L_{n}$ and $\mathbb{Z}_{n}$ remain unchanged and

$$
[i]_{n} * j=\left\{\begin{array}{ll}
i+j & \text { if } i+j<n \\
i+j-n & \text { otherwise, }
\end{array} \quad j *[i]_{n}=j .\right.
$$

Denote the resulting groupoid by $L_{n} \cup \mathbb{Z}_{n}$.

(ii) Let $\mathbb{Z}_{n}^{\prime}=\left\{\left[0^{\prime}\right]_{n}, \ldots,\left[(n-1)^{\prime}\right]_{n}\right\}$ with the usual operation for $\mathbb{Z}_{n}$ and $\mathbb{Z}_{n}^{\prime}$, and

$$
[m]_{n} *\left[k^{\prime}\right]_{n}=\left[(m+k)^{\prime}\right]_{n}, \quad\left[k^{\prime}\right]_{n} *[m]_{n}=\left[k^{\prime}\right]_{n} .
$$

Denote the resulting groupoid by $\mathbb{Z}_{n}^{\prime} \cup \mathbb{Z}_{n}$.

Then $L_{n} \cup \mathbb{Z}_{n} \cong \mathbb{Z}_{n}^{\prime} \cup \mathbb{Z}_{n} \cong L G_{n}$ and $L G_{n}$ is not cryptic.

Proof. The verification is straightforward. We note only that $\left([0]_{n},[1]_{n}\right) \in \mathcal{H}$ and $[0]_{n} * 0=0$ but $[1]_{n} * 0=1$ and $(0,1) \notin \mathcal{H}$, so $L G_{n}$ is not cryptic.

Lemma 6.2. Let $S \in\left\{\Gamma, \Gamma_{\ell}, \Gamma_{\ell r s}\right\}, k \geq 2$, and let $k$ divide $\ell$. For every $m \in \mathbb{Z}$, let $\bar{m}$ satisfy

$$
0 \leq \bar{m}<k, \quad m \equiv m^{\prime}(\bmod k) .
$$

Define $\alpha$ by

$$
\alpha m=\overline{m+1} \quad(0 \leq m<k)
$$


give $I_{k}$ the left zero multiplication, and set

$$
\alpha^{n} * m=\alpha^{n} m, \quad m * \alpha^{n}=m \quad(0 \leq m, n<k) .
$$

Then $\langle\alpha\rangle \cup I_{k} \cong L G_{k}$ and the mapping

$$
\chi:\left\{\begin{array}{l}
b^{n} \mapsto \alpha^{n} \\
(m, g, n) \mapsto \bar{m}
\end{array}\right.
$$

is a homomorphism of $S$ onto $L G_{k}$.

Proof. Note that $k$ divides $\ell$ and $\ell$ divides $s$. The argument consists of a straightforward verification.

THEOREM 6.3. The following conditions on $S$ are equivalent.

(i) $\quad S \in \mathcal{B G}$.

(ii) $S$ has no subsemigroups isomorphic to any $\Gamma, \Gamma_{\ell}, \Gamma_{\ell}^{\prime}, \Gamma_{\ell r s}$ for $\ell+r>2$.

(iii) $S$ has no divisors isomorphic to $L G_{p}$ or $R G_{p}$ for any prime $p$.

(iv) If $\chi: \Gamma_{\infty} \rightarrow S$ is a homomorphism, then the image of $\chi$ is a semilattice of one or two cyclic groups.

Proof. (i) implies (iii). This follows directly from Lemma 6.1 and its dual.

(iii) implies (ii). This is a direct consequence of Lemma 6.2 and its dual.

(ii) implies (i). Let $a \in E(S)$ and $b \in S$ satisfy $a<b^{0}$ and let $T$ be the completely regular subsemigroup of $S$ generated by the set $\{a, b\}$. By [4, Theorem 6.3], $T$ is isomorphic to one of the semigroups $\Gamma, \Gamma_{\ell}, \Gamma_{\ell}^{\prime}, \Gamma_{\ell r s}$ and the hypothesis implies that $r=s=1$. But then $a b=b a$. It remains to apply [3, Theorem 3.3] to see that $S \in \mathcal{B G}$.

(ii) implies (iv). Let $T=\Gamma_{\infty} \chi$. Then $T$ is generated by some set $\{a, b\}$ with $a \leq b^{0}$. If $a=b^{0}$, then $T=\langle b\rangle$ and is thus a cyclic group. If $a<b^{0}$, then in view of [4, Theorem 6.3], the hypothesis implies that $T \cong \Gamma_{11 s}$ for some $s$, and $T$ is a semilattice of two cyclic groups.

(iv) implies (ii). By [4, Theorem 6.3], the homomorphic images $\Gamma_{\infty} \chi$ having two components are of the form $\Gamma, \Gamma_{\ell}, \Gamma_{\ell}^{\prime}, \Gamma_{\ell r s}$. Among these only $\Gamma_{11 s}$ is a semilattice of two cyclic groups. Hence $S$ cannot have subsemigroups isomorphic to $\Gamma, \Gamma_{\ell}, \Gamma_{\ell}^{\prime}$, and $\Gamma_{\ell r s}$ with $\ell+r>2$.

The equivalence of parts (i) and (iii) in the above theorem is Rasin's theorem (see [6, Theorem V.7.6]), for which the above argument provides a new proof. In the case where each $\mathcal{D}$-class of $S$ contains only a finite number of $\mathcal{H}$-classes, the main result in [3, Theorem 8.1] essentially asserts the equivalence of parts (i) and (ii) in Theorem 6.3 (with suitable modification and in different notation).

For the calculation of $\bigvee_{p} \mathcal{L}_{p}$, we will need the following construction.

Denote by $L_{\infty}$ the left zero semigroup on the set $L_{\infty}=\left\{n^{\prime} \mid n \in \mathbb{Z}\right\}$. On $L G_{\infty}=$ $L_{\infty} \cup \mathbb{Z}$ define a product by

$$
m * n^{\prime}=(m+n)^{\prime}, \quad n^{\prime} * m=n^{\prime},
$$

and let $L_{\infty}$ and $\mathbb{Z}$ retain their multiplication. 
Lemma 6.4. $L G_{\infty}$ is a completely regular semigroup. For any $n>1$, the mapping

$$
\chi_{n}: \begin{cases}m \mapsto[m]_{n} & (m \in \mathbb{Z}) \\ m^{\prime} \mapsto\left[m^{\prime}\right]_{n} & \left(m^{\prime} \in L_{\infty}\right)\end{cases}
$$

is a homomorphism of $L G_{\infty}$ onto $\mathbb{Z}_{n}^{\prime} \cup \mathbb{Z}$ (see Lemma 6.1).

Proof. Straightforward verification will show that the multiplication in $L G_{\infty}$ is associative, whence it obviously follows that $L G_{\infty}$ is completely regular. Comparing the above product for $L G_{\infty}$ with Lemma 6.1(ii) makes it clear that $\chi$ has the properties claimed.

By duality, we define $R_{\infty}$ and $R G_{\infty}$.

THeOREM 6.5. For every prime $p$, let $\mathcal{L}_{p}=\left\langle L G_{p}\right\rangle$ and $\mathcal{R}_{p}=\left\langle R G_{p}\right\rangle$.

(i) $\mathcal{L}_{p}=\mathcal{L} \mathcal{R} O \mathcal{A}_{p}, \mathcal{R}_{p}=\mathcal{R} \mathcal{R} O \mathcal{A}_{p}$.

(ii) $\mathcal{L}_{p}$ and $\mathcal{R}_{p}$ are minimal non- $\mathcal{B G}$ varieties.

(iii) Every non- $\mathcal{B G}$ variety contains either $\mathcal{L}_{p}$ or $\mathcal{R}_{p}$ for some prime $p$.

(iv) For $\mathcal{V} \in\{\mathcal{L}, \mathcal{R}\}$ and primes $p$ and $q$, if $\mathcal{V}_{p}=\mathcal{V}_{q}$, then $p=q$. Also, $\mathcal{L}_{p} \neq \mathcal{R}_{q}$ for all primes $p$ and $q$.

Let $\mathcal{L}_{\infty}=\left\langle L G_{\infty}\right\rangle$ and $\mathcal{R}_{\infty}=\left\langle R G_{\infty}\right\rangle$.

(v) $\mathcal{L}_{\infty}=\bigvee_{p} \mathcal{L}_{p}=\mathcal{L} \mathcal{R} O \mathcal{A}=\left[(a x a)^{2} x a y=a x(a x a)^{2} y a^{0}\right]$,

$\mathcal{R}_{\infty}=\bigvee_{p} \mathcal{R}_{p}=\mathcal{R} \mathcal{R O A}=\left[\operatorname{yax}(a x a)^{2}=a^{0} y(a x a)^{2} x a\right]$.

(vi) $\mathcal{L}_{\infty} \vee \mathcal{R}_{\infty}=\mathcal{R O A}=\left\langle L G_{\infty} \times R G_{\infty}\right\rangle=\left[\operatorname{bax}(a x a)^{0} y b=b(a x a)^{0} x a b^{0} y b\right]$.

Proof. The first part of part (i) was proved in [2, Theorem 6.4(v)]; the second is its dual. Parts (ii) and (iii) are from [6, Corollary V.7.7].

(iv) Subgroups of semigroups in $\mathcal{L}_{p}$ are abelian groups whose exponents divide $p$. If $\mathcal{V}_{p}=\mathcal{V}_{q}$, then both $p$ divides $q$ and $q$ divides $p$ so that $p=q$. Moreover, $\mathcal{L}_{p}$ contains all left zero semigroups and only the trivial right zero semigroup; a dual statement is valid for $\mathcal{R}_{q}$ and thus $\mathcal{L}_{p} \neq \mathcal{R}_{q}$.

(v) We consider the first part; the second part is its dual. Define a mapping by

$$
\chi: \begin{cases}m \mapsto\left([m]_{p}\right)_{p \in \mathbb{P}} & (m \in \mathbb{Z}) \\ m^{\prime} \mapsto\left(\left[m^{\prime}\right]_{p}\right)_{p \in \mathbb{P}} & \left(m^{\prime} \in L_{\infty}\right) .\end{cases}
$$

It follows from Lemma 6.4 that $\chi$ is a homomorphism of $L G_{\infty}$ into $\prod_{p}\left(L_{p} \cup \mathbb{Z}_{p}\right)$. If $m \chi=n \chi$, then taking a prime $p$ such that $p>m, n$, we get $m=n$; similarly $m^{\prime} \chi=n^{\prime} \chi$ yields $m^{\prime}=n^{\prime}$. We cannot have $m \chi=n^{\prime} \chi$. Therefore $\chi$ is injective and thus an embedding. But then $\mathcal{L}_{\infty}=\bigvee_{p} \mathcal{L}_{p}$; see [6, Lemma I.8.11].

Since $L G_{p} \in \mathcal{L} \mathcal{R O A}$, we obtain $\mathcal{L}_{p} \subseteq \mathcal{L} \mathcal{R O A}$ and thus $\bigvee_{p} \mathcal{L}_{p} \subseteq \mathcal{L} \mathcal{R} O \mathcal{A}$. One may extract easily from the proof of [2, Theorem $6.4(\mathrm{v})]$ that

$$
\mathcal{L} \mathcal{R O} \subseteq\left[(a x a)^{2} x a y=a x(a x a)^{2} y a^{0}\right] \subseteq \mathcal{L}_{\infty}
$$

we omit the details. 
(vi) In view of part (v), for the first equality, it suffices to prove that

$$
\mathcal{L} O \mathcal{A} \vee \mathcal{R} O \mathcal{A}=\mathcal{R} O \mathcal{A} \text {. }
$$

The inclusion $\mathcal{L} O \mathcal{A} \vee \mathcal{R} O \mathcal{A} \subseteq \mathcal{R O A}$ is trivial, and the reverse inclusion follows from the implication (i) $\Rightarrow$ (iii) of [6, Theorem V.3.3].

In view of part (v) and equality (6.2), we obtain

$$
\mathcal{R O A}=\left\langle L G_{\infty}\right\rangle \vee\left\langle R G_{\infty}\right\rangle \subseteq\left\langle L G_{\infty} \times R G_{\infty}\right\rangle \subseteq \mathcal{R O A}
$$

and equality prevails.

By [6, Theorem V.3.3 and Proposition II.7.2], a basis for identities of $\mathcal{R O} \mathcal{A}$ is given by

$$
\text { axya }=a x a^{0} y a, \quad a x(a x a)^{0}=(a x a)^{0} x a .
$$

Assume the validity of the identity in (6.1). For $a=x^{0}$, we get $b x y b=b x b^{0} y b$, and for $b=y=a^{0}$, we obtain $a x(a x a)^{0}=(a x a)^{0} x a$, so (6.3) holds. Conversely, obviously the identities in (6.3) imply those in (6.1).

\section{Varieties $\mathcal{G}, \mathcal{R e G}, \mathcal{N} O, O B G$, and $L O B G$}

These varieties can be obtained as suitable intersections of $C \mathcal{S}, \mathcal{S G}, \mathcal{N B G}, O, L O$, and $\mathcal{B G}$. The first three of these were treated in Section 3, and the next three in Sections 4-6. The type of information we provided in those sections can be treated for the varieties in the title of this section in a unified way. Hence we change the style of presentation by treating these varieties in a unified way, and in particular, heavily using information from previous sections.

We start our discussion with a lemma which has the desired unifying effect.

Lemma 7.1. Let $\mathcal{U}, \mathcal{V} \in \mathcal{L}(C \mathcal{R})$.

(i) Let $\mathbf{U} \subseteq \mathcal{L}(C \mathcal{R})$ be such that:

(a) $X \in \mathbf{U} \Rightarrow \mathcal{X} \nsubseteq \mathcal{U}$;

(b) $\boldsymbol{y} \in \mathcal{L}(C \mathcal{R}), \boldsymbol{Y} \subset \mathcal{X} \in \mathbf{U} \Rightarrow \mathcal{Y} \subseteq \mathcal{U} \cap \mathcal{V}$;

(c) $\mathcal{W} \in \mathcal{L}(C \mathcal{R}), \mathcal{W} \nsubseteq \mathcal{U} \Rightarrow \mathcal{W}$ contains a member of $\mathbf{U}$.

(ii) Let $\mathbf{V} \subseteq \mathcal{L}(C \mathcal{R})$ have analogous properties.

Then $\mathbf{U} \cup \mathbf{V}$ is a set of minimal non- $\mathcal{U} \cap \mathcal{V}$ varieties and each non- $\mathcal{U} \cap \mathcal{V}$ variety contains a member of $\mathbf{U} \cup \mathbf{V}$.

Proof. (a) Let $\mathcal{X} \in \mathbf{U} \cup \mathbf{V}$. We may assume that $\mathcal{X} \in \mathbf{U}$. Then $\mathcal{X} \nsubseteq \mathcal{U}$ and thus $\mathcal{X} \nsubseteq \mathcal{U} \cap \mathcal{V}$.

(b) Let $\mathcal{Y}$ be a proper subvariety of $\mathcal{X}$ where $\mathcal{X} \in \mathbf{U} \cup \mathbf{V}$. Again we may suppose that $\mathcal{X} \in \mathbf{U}$. The hypothesis implies that $\mathcal{Y} \in \mathcal{U} \cap \mathcal{V}$.

(c) Let $\mathcal{W} \in \mathcal{L}(C \mathcal{R})$ be such that $\mathcal{W} \nsubseteq \mathcal{U} \cap \mathcal{V}$. Then either $\mathcal{W} \nsubseteq \mathcal{U}$ or $\mathcal{W} \nsubseteq \mathcal{V}$. Again we may assume that $\mathcal{W} \nsubseteq \mathcal{U}$. By hypothesis there exists $\mathcal{Z} \in \mathbf{U}$ such that $\mathcal{Z} \subseteq \mathcal{W}$. But then $\mathcal{Z} \in \mathbf{U} \cup \mathbf{V}$. 
Observe that in Lemma 7.1 conditions (a), part of (b), and (c), in view of Lemma 3.1, mean that $\mathbf{U}$ above is the set of all minimal non- $\mathcal{U}$ varieties, and that every non- $\mathcal{U}$ variety contains a minimal non- $\mathcal{U}$ variety.

In the special cases below, we have a stronger property than (b), namely

$$
\mathcal{Y} \subset \mathcal{X} \in \mathbf{U} \Rightarrow \mathcal{Y} \subseteq \mathcal{U}, \mathcal{X} \subseteq \mathcal{V} .
$$

For the special cases of $\mathcal{U}$ and $\mathcal{V}$, we can easily check that conditions in Lemma 7.1 are satisfied, thereby obtaining the statement for $\mathcal{U} \cap \mathcal{V}$.

For $\mathcal{G}=\mathcal{C S} \cap \mathcal{S G}$, see Theorem 3.6 parts (i) and (ii). We have $C \mathcal{S} \sim \mathcal{S}$ and $\mathcal{S G} \sim \mathcal{L Z}, \mathcal{R Z}$, where $\mathcal{S} \subset \mathcal{S G}$ and $\mathcal{L Z}, \mathcal{R Z} \subset \mathcal{C S}$.

For $\mathcal{R} e \mathcal{G}=\mathcal{C S} \cap \mathcal{O}$, see Theorems 3.6(i) and 4.4(i). We have $\mathcal{C S} \sim \mathcal{S}$ and $\mathcal{O} \sim$ $C \mathcal{S} \mathcal{A}_{p}$, where $\mathcal{S} \subset O$ and $C \mathcal{S} \mathcal{A}_{p} \subset C \mathcal{S}$.

For $\mathcal{N O}=\mathcal{N} \mathcal{B G} \cap O$, see Theorems 3.6(iv) and 4.4(i). We have $\mathcal{N B G} \sim$ $\mathcal{L R B}, \mathcal{R R B}$ and $O \sim C \mathcal{S} \mathcal{A}_{p}$, where $\mathcal{L} \mathcal{R B}, \mathcal{R} \mathcal{R} \subset O$ and $C \mathcal{S A} \mathcal{A}_{p} \subset \mathcal{N} \mathcal{B G}$.

For $O \mathcal{B G}=\mathcal{B G} \cap O$, see Theorems 6.5(i) and 4.4(i). We have $\mathcal{B G} \sim$ $\mathcal{L} O \mathcal{A}_{p}, \mathcal{R} O \mathcal{A}_{p}$ and $O \sim C \mathcal{S} \mathcal{A}_{p}$, where $\mathcal{L} \mathcal{R} O \mathcal{A}_{p}, \mathcal{R} O \mathcal{A}_{p} \subset O$ and $C \mathcal{S} \mathcal{A}_{p} \subset \mathcal{B G}$.

For $L O B G=\mathcal{B G} \cap L O$, see Theorems 6.5(i) and 5.3(i). We have $\mathcal{B G} \sim$ $\mathcal{L} O \mathcal{A}_{p}, \mathcal{R} \mathcal{R} O \mathcal{A}_{p}$ and $L O \sim \mathcal{D}_{p}^{1}$, where $\mathcal{L} \mathcal{R} O \mathcal{A}_{p}, \mathcal{R} O \mathcal{A}_{p} \subset L O$ and $\mathcal{D}_{p}^{1} \subset \mathcal{B G}$.

In all the cases considered, if $\mathcal{U} \sim \mathfrak{U}$ and $\mathcal{V} \sim \mathfrak{B}$, then $\mathcal{U} \cap \mathcal{V} \sim \mathfrak{U} \cap \mathfrak{B}$.

Next we compute the join of the varieties in $\mathfrak{U} \cup \mathfrak{B}$. First we make a digression which will be used in the proof of the next theorem. The following is [7, Corollary 5.3] in the present notation.

FACT 7.2. Let $\mathcal{V} \in[\mathcal{R B}, L O B G]$ and let

$$
\mathcal{V} \cap \mathcal{C S}=\left[u_{\alpha}=v_{\alpha}\right]_{\alpha \in A}, \quad \mathcal{V} \cap \mathcal{B}=\left[r=s, a^{2}=a\right] .
$$

Then a basis for identities in $\mathcal{V}$ is

$$
\begin{gathered}
u_{\alpha}^{*}=v_{\alpha}^{*}, \quad(\alpha \in A), \\
r^{0}=s^{0}, \\
(x r y)^{0}=(x s y)^{0}, \\
\left(x a^{2} y\right)^{0}=(x a y)^{0}, \\
(x a x)^{0}(x b x)^{0}=\left((x a x)^{0}(x b x)^{0}\right)^{0} .
\end{gathered}
$$

According to [7, Theorem 4.4], $\left[u_{\alpha}^{*}=v_{\alpha}^{*}\right]_{\alpha \in A}$ is the greatest member of $\mathcal{L}(C \mathcal{R})$ whose intersection with $C \mathcal{S}$ equals $\left[u_{\alpha}=v_{\alpha}\right]_{\alpha \in A}$. The words $u_{\alpha}^{*}$ and $v_{\alpha}^{*}$ are constructed from $u_{\alpha}$ and $v_{\alpha}$, respectively, in an explicit way. We show next that (7.2) and (7.4) are redundant.

For any word $w$, let $h(w)$ denote the head (the first letter) of $w$, and $t(w)$ denote the tail (the last letter) of $w$.

(1) The hypothesis that $\mathcal{R B} \subseteq \mathcal{V}$ implies that $h(r)=h(s)$ and $t(r)=t(s)$. By setting $x=(h(r))^{0}$ and $y=(t(r))^{0}$, the equation in (7.3) implies that in (7.2). 
(2) According to the proofs of [6, Corollaries II.7.5 and II.8.6], the identity in (7.5) defines the variety $L O B G$ of locally orthodox cryptogroups.

(3) Since $L O B G \subseteq B \mathcal{B}$, and the latter obviously implies (7.4), we may omit (7.4) as well.

(4) There remain relations (7.1), (7.3), and $L O B G$ in Fact (7.2). In the special case that $C \mathcal{S} \subseteq \mathcal{V}$, we can also omit (7.1) since $(C S)^{*}=C \mathcal{R}$.

FACT 7.3. The varieties $C \mathcal{S}, \mathcal{B}$, and $O$ are neutral in $\mathcal{L}(C \mathcal{R})$.

Proof. See [8, Proposition 2.2 and Corollary 2.9].

We are now ready for the desired result.

Theorem 7.4. For $\mathcal{V} \in \mathcal{L}(C \mathcal{R})$, denote by $\mathcal{V}^{\vee}$ the join of all minimal non- $\mathcal{V}$ varieties (if $\mathcal{V}$ has any).

(i) $\mathcal{G}^{\vee}=\mathcal{S} \vee \mathcal{R B}=\mathcal{N B}$.

(ii) $\mathcal{R} e \mathcal{G}^{\vee}=\mathcal{S} \vee C S \mathcal{A}=\mathcal{N B} \mathcal{A}$.

(iii) $\mathcal{N} O^{\vee}=\mathcal{R} e \mathcal{B} \vee C S \mathcal{A}=L O \cap \mathcal{R B G} \cap H \mathcal{A}$.

(iv) $O \mathcal{B G}^{\vee}=\mathcal{R O A} \vee C S \mathcal{A}=L O \cap(\mathcal{R O})^{T} \cap H \mathcal{A}$.

(v) $L O \mathcal{B G}^{\vee}=\mathcal{R O A} \vee \mathcal{D}_{\infty}^{1}$.

PRoof. In view of the preceding comments, we express each of the varieties above as the intersection $\mathcal{U} \cap \mathcal{V}$ where for $\mathcal{U}$ and $\mathcal{V}$ we have computed the join of minimal non- $\mathcal{U}$ and non- $\mathcal{V}$ varieties, and determine their join. Hence the first equality in each part follows from the results in the preceding four sections.

We now prove the second equality in each part.

(i) It is well known that $\mathcal{S} \vee \mathcal{R B}=\mathcal{N B}$.

(ii) This forms part of [6, Theorem IV.1.12(iii)].

(iii) Let $\mathcal{V}=\operatorname{Re} \mathcal{B} \vee C S \mathcal{A}$. We set up the situation in order to apply Fact 7.2. First note that $\mathcal{R B} \subseteq \mathcal{V} \subseteq L O \mathcal{B G}$. Fact 7.3 asserts that $C \mathcal{S}$ is a neutral element of $\mathcal{L}(C \mathcal{R})$, and thus

$$
\mathcal{V} \cap \mathcal{C S}=(\mathcal{R} e \mathcal{B} \cap C \mathcal{S}) \vee(C \mathcal{S A} \cap C \mathcal{S})=C \mathcal{S A} .
$$

By the same reference, also $\mathcal{B}$ is neutral in $\mathcal{L}(C \mathcal{R})$, and hence

$$
\mathcal{V} \cap \mathcal{B}=(\mathcal{R} e \mathcal{B} \cap \mathcal{B}) \vee(C \mathcal{S A} \cap \mathcal{B})=\mathcal{R} e \mathcal{B}
$$

If $\mathcal{U}=\left[u_{\alpha}=v_{\alpha}\right]_{\alpha \in A} \in \mathcal{L}(C \mathcal{R})$, we write $\mathcal{U}^{*}=\left[u_{\alpha}^{*}=v_{\alpha}^{*}\right]_{\alpha \in A}$; see Fact 7.2 and the comments following it. Clearly $\mathcal{A}^{*}=H \mathcal{A}$. By the modified version of Fact 7.2 , we obtain

$$
\mathcal{V}=L O \mathcal{B G} \cap\left[(\text { uaxyav })^{0}=(\text { uaxayav })^{0}\right] \cap H \mathcal{A} .
$$

For $u=v=a^{0}$, the identity in (7.6) yields $(a x y a)^{0}=(\text { axaya })^{0}$. Conversely, assume the validity of the last identity. By [6, Proposition V.4.4], it determines the variety $\mathcal{R} \mathcal{B G}$. Thus we can use the cryptic property to get

$$
(\text { uaxyav })^{0}=\left(u(\text { axya })^{0} v\right)^{0}=\left(u(\text { axaya })^{0} v\right)^{0}=(\text { uaxayav })^{0} .
$$


We have proved that the middle variety in (7.6) equals $\mathcal{R B G}$. Now (7.6) implies that

$$
\mathcal{V}=L O \mathcal{B G} \cap \mathcal{R B G} \cap H \mathcal{A}=L O \cap \mathcal{R B G} \cap H \mathcal{A} .
$$

(iv) For any $\mathcal{U} \in \mathcal{L}(C \mathcal{R})$, by [1, Section 6], $\mathcal{U}^{+}$defined in [7] coincides with $\mathcal{U}^{T}$, the upper end of the trace class of $\mathcal{U}$. Recall that

$$
C \mathcal{U}=\{S \in C \mathcal{R} \mid C(S) \in \mathcal{U}\}
$$

where $C(S)$ is the core of $S$ (the subsemigroup of $S$ generated by $E(S)$ ). Now [7, Proposition 3.5] implies that $O^{T}=C \mathcal{B G}$. Recall the notation $\mathcal{U}^{*}$ in part (iii).

Let $\mathcal{V}=\mathcal{R O A} \vee C S \mathcal{A}$. Then $\mathcal{V} \subseteq L O \cap C \mathcal{B G}$ and [7, Theorem 5.1] implies that

$$
\mathcal{V}=L O \cap(\mathcal{V} \cap O)^{T} \cap(\mathcal{V} \cap C S)^{*}
$$

By Fact 7.3, the variety $O$ is neutral in $\mathcal{L}(C \mathcal{R})$ and thus

$$
\mathcal{V} \cap \mathcal{O}=(\mathcal{R O A} \cap O) \vee(C \mathcal{A} \cap O)=\mathcal{R} O \mathcal{A} \vee \mathcal{R e c} \mathcal{A}=\mathcal{R} O \mathcal{A} .
$$

By the same reference, also $C \mathcal{S}$ is neutral in $\mathcal{L}(C \mathcal{R})$ so that

$$
\mathcal{V} \cap C \mathcal{S}=(\mathcal{R O A} \cap C \mathcal{S}) \vee(C \mathcal{A} \cap C \mathcal{S})=C \mathcal{S A} .
$$

As in part (iii), we have $(C \mathcal{S A})^{*}=H \mathcal{A}$. By (7.7), (7.8), (7.9), and [5, Theorem 6.2], we get

$$
\mathcal{V}=L O \cap(\mathcal{R O A})^{T} \cap H \mathcal{A}=L O \cap(\mathcal{R O})^{T} \cap(H \mathcal{A})^{T} \cap H \mathcal{A}=L O \cap(\mathcal{R O})^{T} \cap H \mathcal{A} .
$$

We may write

$$
\mathcal{N B}=L O \cap \mathcal{N B} \cap H \mathcal{A}, \quad \mathcal{N} \mathcal{B} \mathcal{A}=L O \cap \mathcal{N B G} \cap H \mathcal{A} .
$$

Comparing this with parts (iii) and (iv) of Theorem 7.4, we begin to see a pattern.

In order to characterize the varieties in the title of this section in terms of a homomorphism, we need the following construction.

Let $D_{\infty}^{(e)}$ be the semigroup $D_{\infty}$ with an element $e$ adjoined with products

$$
e=e^{2}, \quad(m, k, n) e=(m, k, n)(0,0,0), \quad e(m, k, n)=(0,0,0)(m, k, n) .
$$

Then $D_{\infty}^{(e)}$ is a retract extension of $D_{\infty}$ with retraction

$$
\psi:\left\{\begin{array}{l}
(m, k, n) \mapsto(m, k, n) \\
e \mapsto(0,0,0) .
\end{array}\right.
$$

We may say that we 'inflated' the element $(0,0,0)$ of $D_{\infty}$ by an idempotent $e$.

THEOREM 7.5.

(i) $S \in \mathcal{G}$ if and only if whenever $\chi: L_{2} \times Y_{2} \times R_{2} \rightarrow S$ is a homomorphism, then the image of $\chi$ is trivial. 
(ii) $S \in \mathcal{R e G}$ if and only if whenever $\chi: D_{\infty}^{(e)} \rightarrow S$ is a homomorphism, then the image of $\chi$ is a small rectangular band.

(iii) $S \in \mathcal{N O}$ if and only if whenever $\chi: D_{\infty}^{1} \rightarrow S$ is a homomorphism, then the image of $\chi$ is a one- or two-element semilattice.

Proof. (i) Straightforward.

(ii) Necessity. The image of $\chi$ is a subsemigroup of a rectangular group and thus must itself be a rectangular group. Clearly $e \chi=(0,0,0) \chi$ and $\left.\chi\right|_{D_{\infty}}$ is a homomorphism of $D_{\infty}$ into $S$. By Lemma 4.1, its image must be a small rectangular band. But $D_{\infty}^{(e)} \chi=D_{\infty} \chi$ so the same holds for $D_{\infty}^{(e)}$.

Sufficiency. The argument is by contrapositive. Hence assume that $S$ is not a rectangular group. First assume that $S$ is completely simple. Then $S$ is not orthodox, and by Theorem 4.2, there is a homomorphism $\varphi: D_{\infty} \rightarrow S$ whose image is not a small rectangular band. Using the retraction $\psi$ in (7.10), the composition $\psi \varphi: D_{\infty}^{(e)} \rightarrow S$ is a homomorphism whose image is not a small rectangular band. Suppose next that $S$ is not completely simple. Then it contains idempotents $f>g$. Defining a mapping

$$
\chi:\left\{\begin{array}{l}
e \mapsto f \\
(m, k, n) \mapsto g \quad\left((m, k, n) \in D_{\infty}\right),
\end{array}\right.
$$

we obtain a homomorphism $\chi: D_{\infty}^{(e)} \rightarrow S$ whose image is a nontrivial semilattice, and is thus not a rectangular band.

(iii) From Lemma 5.1, we deduce that homomorphic images of $D_{\infty}^{1}$ are, up to isomorphism,

$$
D_{\infty}^{1}, D_{n}^{1} \text { for } n>1,\left(L_{2} \times R_{2}\right)^{1}, L_{2}^{1}, R_{2}^{1}, Y_{2},\{0\} .
$$

Only the last two of these are normal orthogroups. The assertion now follows from Theorem 5.2.

We do not have the corresponding statements for $O B G$ and $L O B G$.

\section{Review}

Besides the intrinsic interest of some of our results as isolated statements, their comparison may even be more useful if we have in mind some general patterns. For this purpose, we now tabulate essential parts of our results for all varieties considered.

In Table 1 we exhibit the main results of Section 3. These are simple statements whose proofs are essentially omitted, and most of which are folklore.

Table 2 is a review of cases studied in Sections 4-6. This is the main part of the paper.

Table 3 reviews the varieties which can be obtained as intersections of some previously treated. These results rely heavily on previously proved statements, and are only briefly summarized.

We have not stated bases for these varieties since they follow immediately from the glossary of varieties and their bases are in Section 2. 
TABLE 1. Varieties from Section 3.

\begin{tabular}{lcccc}
\hline Variety $\mathcal{V}$ & $C \mathcal{S}$ & $\mathcal{S}$ & $\mathcal{S G}$ & $\mathcal{N B G}$ \\
\hline See Theorem 3.6 and Proposition & 3.2 & 3.3 & 3.4 & 3.5 \\
Forbidden subsemigroups & $Y_{2}$ & $L_{2}, \mathbb{Z}, \mathbb{Z}_{n}, R_{2}$ & $L_{2}, R_{2}$ & $L_{2}^{1}, R_{2}^{1}$ \\
Forbidden divisors & $Y_{2}$ & $L_{2}, \mathbb{Z}_{p}, R_{2}$ & $L_{2}, R_{2}$ & $L_{2}^{1}, R_{2}^{1}$ \\
Minimal non- $\mathcal{V}$ varieties & $\mathcal{S}$ & $\mathcal{L Z}, \mathcal{A}_{p}, \mathcal{R Z}$ & $\mathcal{L Z}, \mathcal{R Z}$ & $\mathcal{L} \mathcal{R} \mathcal{R}, \mathcal{R B}$ \\
Their join & $\mathcal{S}$ & $\operatorname{Rec} \mathcal{A}$ & $\mathcal{R B}$ & $\mathcal{R e} \mathcal{B}$ \\
Its generator & $Y_{2}$ & $L_{2} \times \mathbb{Z} \times R_{2}$ & $L_{2} \times R_{2}$ & $\left(L_{2} \times R_{2}\right)^{1}$ \\
Test semigroup & $Y_{2}$ & $L_{2} \times \mathbb{Z} \times R_{2}$ & $L_{2} \times R_{2}$ & $\left(L_{2} \times R_{2}\right)^{1}$ \\
Its image & trivial & trivial & trivial & trivial or $Y_{2}$ \\
\hline
\end{tabular}

TABle 2. Varieties from Sections 4-6.

\begin{tabular}{lccc}
\hline Variety $\mathcal{V}$ & $O$ & $L O$ & $\mathcal{B G}$ \\
\hline See Theorems & 4.2 and 4.4 & 5.2 and 5.3 & 6.3 and 6.5 \\
Forbidden subsemigroups & $D_{n}, D_{\infty}, n>1$ & $D_{n}^{1}, D_{\infty}^{1}, n>1$ & $\Gamma, \Gamma_{\ell}, \Gamma_{\ell}^{\prime}, \Gamma_{\ell r s}$ \\
Forbidden divisors & $D_{p}$ & $D_{p}^{1}$ & $L G_{p}, R G_{p}$ \\
Minimal non- $\mathcal{V}$ varieties & $C \mathcal{S} \mathcal{A}_{p}$ & $\mathcal{D}_{p}^{1}$ & $\mathcal{L} \mathcal{R} O \mathcal{A}_{p}, \mathcal{R} \mathcal{R} O \mathcal{A}_{p}$ \\
Their join & $C \mathcal{A}$ & $\mathcal{D}_{\infty}^{1}$ & $\mathcal{R} O \mathcal{A}$ \\
Its generator & $D_{\infty}$ & $D_{\infty}^{1}$ & $L G_{\infty} \times R G_{\infty}$ \\
Test semigroup & $D_{\infty}$ & $D_{\infty}^{1}$ & $\Gamma_{\infty}$ \\
Its image & Trivial or $L_{2}$ or $R_{2}$ & Trivial or $L_{2}^{1}$ & Semilattice of one or \\
& or $L_{2} \times R_{2}$ & or $R_{2}^{1}$ or $\left(L_{2} \times R_{2}\right)^{1}$ & two cyclic groups \\
\hline
\end{tabular}

TABLE 3. Some intersections of varieties from Sections 3-6.

\begin{tabular}{lccc}
\hline Variety $\mathcal{V}$ & $\begin{array}{l}\text { Join of minimal } \\
\text { non- } \mathcal{V} \text { varieties }\end{array}$ & Test semigroup & Its image \\
\hline See Theorem & 7.4 & 7.5 & 7.5 \\
$\mathcal{G}=C \mathcal{S} \cap \mathcal{S G}$ & $\mathcal{N} \mathcal{B}$ & $L_{2} \times Y_{2} \times R_{2}$ & Trivial \\
$\mathcal{R} e \mathcal{G}=C \mathcal{S} \cap O$ & $\mathcal{N} \mathcal{B A}$ & $D_{\infty}^{(e)}$ & Trivial or $L_{2}$ or $R_{2}$ or $L_{2} \times R_{2}$ \\
$\mathcal{N} O=\mathcal{N} \mathcal{B G} \cap O$ & $L O \cap \mathcal{R B G} \cap H \mathcal{A}$ & $D_{\infty}^{1}$ & Trivial or $Y_{2}$ \\
$O \mathcal{B G}=\mathcal{B G} \cap O$ & $L O \cap(\mathcal{R O})^{T} \cap H \mathcal{A}$ & & \\
$L O B G=B G \cap L O$ & $\mathcal{R O A} \vee \mathcal{D}_{\infty}^{1}$ & & \\
\hline
\end{tabular}




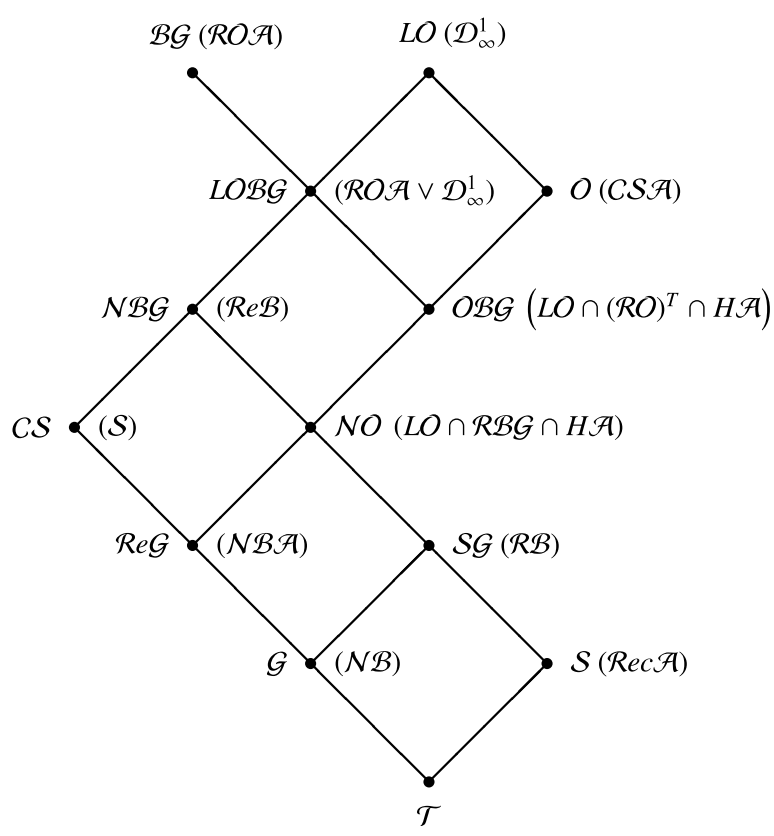

FIGURE 1.

In Figure 1 the vertices are labelled by the varieties $\mathcal{V}$ studied as well as $\mathcal{V}^{\vee}$ in parentheses.

\section{References}

[1] P. R. Jones, 'Mal'cev products of varieties of completely regular semigroups', J. Aust. Math. Soc. Ser. A 42 (1987), 227-246.

[2] M. Petrich, 'Characterizations of certain completely regular varieties', Semigroup Forum 66 (2003), 381-400.

[3] M. Petrich, 'Characterizing cryptogroups with a finite number of $\mathcal{H}$-classes in each $\mathcal{D}$-class by their subsemigroups', Ann. Mat. Pura Appl. (4) 187 (2008), 119-136.

[4] M. Petrich, 'Completely regular monoids with two generators', J. Aust. Math. Soc. 90 (2011), 271-287.

[5] M. Petrich and N. R. Reilly, 'Operators related to $E$-disjunctive and fundamental completely regular semigroups', J. Algebra 134 (1990), 1-27.

[6] M. Petrich and N. R. Reilly, Completely Regular Semigroups (Wiley, New York, 1999).

[7] N. R. Reilly, 'Varieties of completely regular semigroups', J. Aust. Math. Soc. Ser. A 38 (1985), 372-393.

[8] P. G. Trotter, 'Subdirect decompositions of the lattice of varieties of completely regular semigroups', Bull. Aust. Math. Soc. 39 (1989), 343-351.

\section{MARIO PETRICH, 21420 Bol, Brač, Croatia}

